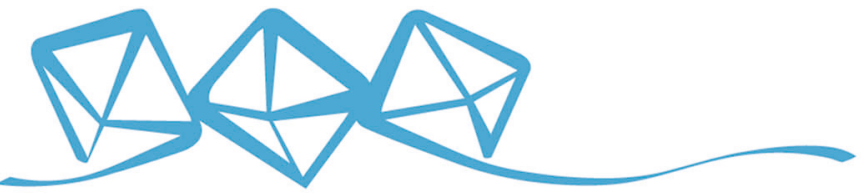 COMMUNICATIONS MATERIALS
}

ARTICLE

Check for updates

https://doi.org/10.1038/s43246-020-00073-3 OPEN

\section{Nanographitic coating enables hydrophobicity in lightweight and strong microarchitected carbon}

\author{
Akira Kudo ${ }^{1} \&$ Federico Bosi (1D ${ }^{2 凶}$
}

Metamaterials that are lightweight, stiff, strong, scalable and hydrophobic have been achieved separately through different materials and approaches, but achieving them in one material is an outstanding challenge. Here, stereolithography and pyrolysis are employed to create carbon microlattices with cubic topology and a strut width of $60-70 \mu \mathrm{m}$, with specific strength and stiffness of up to $468.62 \mathrm{MPa} \mathrm{cm}^{3} \mathrm{~g}^{-1}$ and $14.39 \mathrm{GPa} \mathrm{cm}^{3} \mathrm{~g}^{-1}$ at a density of $0.55 \mathrm{~g} \mathrm{~cm}^{-3}$, higher than existing microarchitected materials and approaching those of the strongest truss nanolattices. Subsequent fast Joule-heating then introduces a hierarchical nanographitic skin that enables hydrophobicity, with a water contact angle of $135 \pm 2^{\circ}$, improving the hydrophilic response of pyrolytic carbon. As the Joule heating induced $\mathrm{sp}^{2}$-hybridization and nano-texturing predominantly affect the strut sheath, the effect on mechanical response is limited to a reduction in the distribution of compressive strength of as-pyrolyzed architectures by $\sim 80 \%$ and the increase of the mean effective stiffness by $\sim 15 \%$. These findings demonstrate a technique to fabricate high strength, low density, and hydrophobic nanographite-coated carbon microlattices.

\footnotetext{
${ }^{1}$ Division of Engineering and Applied Science, California Institute of Technology, Pasadena, CA 91125, USA. ${ }^{2}$ Department of Mechanical Engineering, University College London, London WC1E 7JE, UK. ${ }^{凶}$ email: f.bosi@ucl.ac.uk
} 
S ince its inception and early studies ${ }^{1}$, additive manufacturing has become a powerful engineering tool, capable of creating three-dimensional structures with dimensions ranging from multi-meter scale to submicron by using a variety of materials like metals $^{2-5}$, ceramics ${ }^{6,7}$, glasses ${ }^{8,9}$, polymers ${ }^{10}$, and composites ${ }^{11}$. Through a micro- and nanoscale topological control, advanced applications of 3D-printed architectures include scaffolds ${ }^{12}$, tissue engineering ${ }^{13}$, supercapacitors ${ }^{14}$, solar cells ${ }^{15}$, lightweight metamaterials ${ }^{16-18}$, and many others.

The field of mechanical metamaterials has particularly benefitted from the progress of reduced-scale manufacturing that has allowed the creation of architected materials with superior properties, mutually exclusive in traditional solids ${ }^{19-22}$. Such successful development comprises the realization of extremely deformable lattices constituted of brittle materials ${ }^{23}$, or simultaneously lightweight, stiff and strong architectures that exploit a hierarchical design $^{24}$ and the nanoscale strengthening effect ${ }^{25,26}$. The latter example has been achieved with carbon nanoarchitectures obtained by means of pyrolysis of polymeric lattices fabricated through two-photon lithography, which operates by cross-linking a liquid precursor resin voxel-by-voxel in a relatively slow process that prevents large-scale manufacturing. These carbon nanolattices have achieved the highest specific strength and stiffness (strength and stiffness to density ratio) ever attained for lightweight architected materials with densities below $1.0 \mathrm{~g} \mathrm{~cm}^{-3}$, approaching the theoretical strength of the constituent material through a reduction of dimensions to the critical size of flaw insensitive solids ${ }^{25-27}$. On the other hand, carbon microlattices obtained via self-propagating photopolymer waveguides $^{28}$ and stereolithography (SLA) ${ }^{29}$ show rapid prototyping of meso- and macroscale structures. However, their mechanical properties do not exceed those of traditional bulk materials, with specific strength limited to $53.68 \mathrm{MPa} \mathrm{cm}^{3} \mathrm{~g}^{-1}$, and specific stiffness bounded to $5.79 \mathrm{GPa} \mathrm{cm}^{3} \mathrm{~g}^{-1}$, thus not exploiting the advantages of an architected lattice design through additive manufacturing ${ }^{28}$. Therefore, one of the major challenges in metamaterial design is to simultaneously achieve high compressive mechanical properties and a fast, scalable fabrication.

Most of the studies on carbon architected materials have been focused on the synthesis and mechanical characterization ${ }^{29-34}$. The investigation of properties beyond the mechanical response could pave the pathway to multifunctional material design. Hydrophobicity, controlled through rational design of the lattice micro-texture, can be synergetic to the excellent mechanical performance of additively manufactured carbon lattices. The combination of hydrophobicity and strength in a single resistant material is sought for structural components exposed to extreme environments, to reduce wear and corrosion of lightweight systems in aerospace and maritime engineering, with applications ranging from anti-icing structures ${ }^{35}$ to self-cleaning surfaces ${ }^{36}$. However, a simultaneous demonstration of these features is challenging. Currently, water repellent three-dimensionally fabricated structures are limited to planar arrays of microarchitectures $^{37}$ and soft lattices ${ }^{38}$, which do not guarantee durability and load-bearing capacity.

In this work, we present an approach based on pyrolysis and Joule heating to create lightweight 3D microarchitected carbon that combines superior mechanical performances and considerable hydrophobicity. The former process is mainly responsible for the advanced structural features, while the latter predominately enhances the water contact angle. Through an anisotropic unit cell topology, the specific strength and stiffness reached 468.62 $\mathrm{MPa} \mathrm{cm} \mathrm{g}^{-1}$ and $14.39 \mathrm{GPa} \mathrm{cm}^{3} \mathrm{~g}^{-1}$, outperforming all existing meso- and microlattices and attaining values that approach those of the strongest open-cell nanoarchitected materials developed up to date, despite presenting strut width and length two orders of magnitude larger. We show that Joule heating transforms the mainly amorphous as-pyrolyzed microarchitectures into glassy/ nanographitic carbon core/shell morphologies. We prove that the lattice architecture converts the hydrophilic pyrolytic carbon into a hydrophobic material with a water contact angle of $103 \pm 12^{\circ}$, and the Joule heating induces a porous nanographitic skin that further enhances the contact angle to $135 \pm 2^{\circ}$, thus approaching superhydrophobicity. Since the fast Joule heating process mainly affects the strut surface, the changes in structural response in Joule heated lattices are limited to a reduction of the distribution of compressive strengths by $\sim 80 \%$ while preserving the remarkable mean failure stress, and to an increase of the average effective stiffness by $~ 15 \%$. On the one hand, the heat-induced defect-rich nanoporous surface mitigates scattering of the failure stress and controls the onset of catastrophic failure; on the other hand, it prevents the Joule heated architectures from reaching the highest strength recorded in aspyrolyzed lattices. These results demonstrate a feasible methodology to create nanographite-coated microstructured carbon, with promising applications in extreme environments.

\section{Results}

Additive manufacturing and material characterization of carbon microlattices. The Digital Light Processing (DLP) SLA 3D-printed polymeric lattices were obtained from a transparent PR-48 photoresist resin. Subsequently, they were subjected to pyrolysis that yielded fully dense carbon microlattices, associated with an isotropic 66\% linear and 97\% volumetric shrinkage (Supplementary Fig. 1). The Joule heating process after pyrolysis did not affect the lattice microarchitecture (Fig. 1a, b and Supplementary Fig. 2), which presented cubic unit cells, dimensions of $\sim 200 \mu \mathrm{m}$, strut width of $60-70 \mu \mathrm{m}$ and a density of $0.55 \mathrm{~g} \mathrm{~cm}^{-3}$. Further details on the fabrication process can be found in the "Method" section.

Scanning electron microscopy (SEM) images in Fig. 1c, d show that SLA introduced imperfections in the architected microstructure, designed initially with cubic unit cells with constant beam width. The manufactured lattices presented an orientationdependent strut width, with beams deposited parallelly to the printer platform that resulted $\sim 20 \%$ wider than the ones built along the vertical direction. The relative density was calculated for both types of lattices, showing $\bar{\rho}=0.30 \pm 0.01$ for as-pyrolyzed and $\bar{\rho}=0.28 \pm 0.01$ for Joule-heated microlattices, within the limit of true cellular solids. Furthermore, SEM images at progressive magnification conveyed that the features present in the originallysculpted polymer lattices were preserved throughout the heat treatments (Fig. 1c, d). The 7-9 $\mu \mathrm{m}$-separated grooves that populated each beam constituted the traces of layer-by-layer SLA. The texture of as-pyrolyzed samples appeared smooth, while Joule-heated specimens presented sporadically located micropores, with diameter lower than $2 \mu \mathrm{m}$, and homogeneously distributed nanopores, with diameter smaller than $50 \mathrm{~nm}$ (Fig. 1c, d).

Atomic force microscopy (AFM) characterized changes in the surface roughness introduced by the Joule heating treatment. AFM images at low magnification on $40 \mu \mathrm{m} \times 40 \mu \mathrm{m}$ areas revealed that the groove depth on the surface of as-pyrolyzed samples presented a root mean square (RMS) of $661 \pm 114 \mathrm{~nm}$ (Fig. 2a, left column), while high magnification over $300 \mathrm{~nm} \times 300 \mathrm{~nm}$ regions proved the smoothness of the surface of as-pyrolyzed carbon, with protrusions limited to an RMS value of $5.26 \pm 2.24 \mathrm{~nm}$ (Fig. 2a, right column). Joule heating treatment increased surface roughness to an RMS of $734 \pm 44 \mathrm{~nm}$ and created micropores, visible from low magnification images (Fig. 2b, left column). At the nanoscale, roughness became nearly fourfold, with an RMS value of $19.07 \pm 9.36 \mathrm{~nm}$, as a result of nanopores developed through Joule heating (Fig. 2b, right column). 


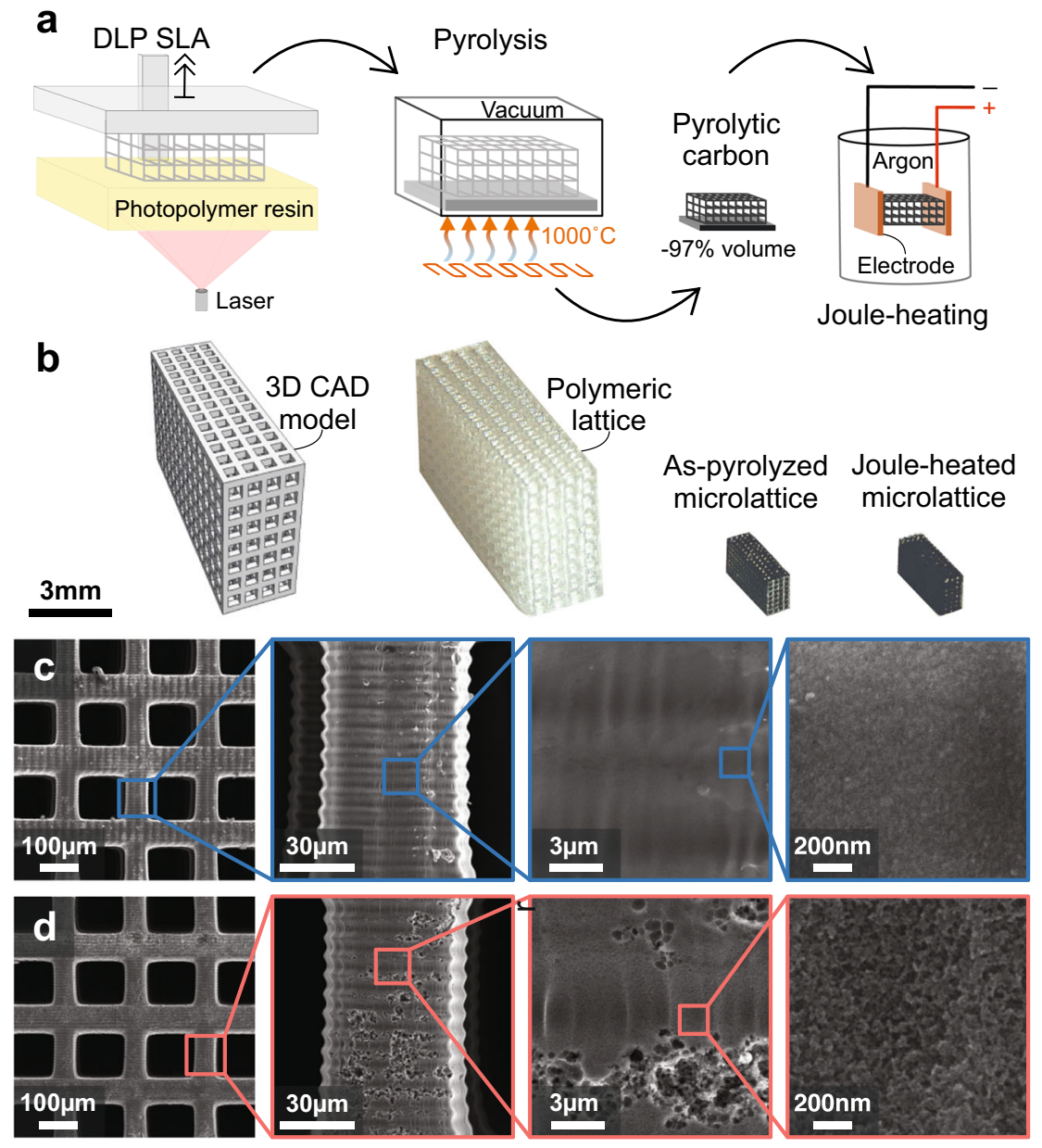

Fig. 1 Fabrication and SEM characterization of as-pyrolyzed and Joule-heated carbon microlattices. a Illustration of the manufacturing process that includes DLP SLA of cubic microarchitectures from photoresist resin, high-temperature pyrolysis up to $1000^{\circ} \mathrm{C}$ under vacuum, and Joule heating in an argon environment. $\mathbf{b}$ Three-dimensional lattice CAD model and optical images of additively manufactured polymeric, pyrolytic carbon and Joule-heated carbon microarchitectures. SEM images of representative as-pyrolyzed (c) and Joule-heated (d) carbon microlattices, showing that the features introduced by $3 \mathrm{D}$-fabrication are orientation-dependent strut width and micrometer groove pattern. Progressive magnification reveals the smooth surfaces of the former and the porous morphology of the latter.

Existing literature on pyrolyzed carbon nano- and microarchitected materials suggests that the atomic-scale structures of nanolattices fabricated by two-photon lithography ${ }^{25}$, microlattices manufactured through self-propagating photopolymer waveguide $^{28}$, and microarchitectures produced by SLA ${ }^{29}$, are all glassy carbon, a class of $\mathrm{sp}^{2}$-hybridized carbon. Figure $3 \mathrm{a}$ and Supplementary Fig. 3 compare Raman spectra collected from the skin and inner core of the beams obtained from both types of microlattices. The spectra indicated a skin-core structure that resembles the morphology of polyacrylonitrile (PAN)-derived carbon fiber ${ }^{39}$, where the outer shell contains more $\mathrm{sp}^{2}$ hybridized carbon and less amorphous carbon than the inner core does. As-pyrolyzed samples presented a moderately $\mathrm{sp}^{2}-$ hybridized surface, evidenced by the distinguished $D$ and $G$ peaks and the indiscernible $2 \mathrm{D}, \mathrm{D}+\mathrm{D}^{\prime}$ and $2 \mathrm{D}^{\prime}$ peaks that form a broad single peak at $2800 \mathrm{~cm}^{-1}$. The internal core of the beams, on the other hand, shows only a shallow split between D and G peaks since the integrated intensity of amorphous carbon component at $1505 \mathrm{~cm}^{-1}$ surpasses that of $\mathrm{G}$ peak ${ }^{40}$. This observation suggests that pyrolyzing the $\mathrm{PR} 48$ photoresist polymer at $1000^{\circ} \mathrm{C}$ produces mainly amorphous carbon with a low degree of $\mathrm{sp}^{2}$-hybridization. In the Joule-heated specimens, although the fraction of $\mathrm{sp}^{2}$-hybridized carbon generally increased throughout the microstructure, the different composition between the surface and the core of the beams remained. The inner core after Joule heating presented a moderately $\mathrm{sp}^{2}$-hybridized carbon, similar to the as-pyrolyzed skin. The surface of the beams, on the other hand, contained features of emerging nanocrystalline graphites. The relative area intensity of $G$ peak to amorphous carbon peak nearly doubled compared to the as-pyrolyzed skin (Supplementary Fig. 4), deepening the valley between $\mathrm{G}$ and $\mathrm{D}$ peaks ${ }^{41-44}$. Furthermore, separate second-order peaks, namely $2 \mathrm{D}\left(2690 \mathrm{~cm}^{-1}\right), \mathrm{D}+\mathrm{D}^{\prime}$ $\left(2930 \mathrm{~cm}^{-1}\right)$ and $2 \mathrm{D}^{\prime}\left(3190 \mathrm{~cm}^{-1}\right)$, visible only in Joule-heated skin, imply the presence of defect-rich graphitic structures ${ }^{41,45,46}$ (Supplementary Fig. 5). The D peak higher than the nominal G peak ( $G$ and $D^{\prime}$ treated as a single peak at $1600 \mathrm{~cm}^{-1}$ ) for this case also suggests nanographites crystallized from the amorphous matrix ${ }^{47}$.

HRTEM imaging visualized the structural changes induced by Joule heating at an atomic scale. Although Joule-heated carbon was still mainly non-crystalline, stacks of graphene sheets became conspicuous, with a distinguishable peak from (002) direction in the Fast Fourier Transformation (FFT) pattern (Fig. 3b, c and Supplementary Fig. 6). Joule-heated carbon locally presented $\sim 10 \mathrm{~nm}$-long clusters of assembled graphene sheets spaced at 3.4-3.7 $\AA$ (Fig. 3d). In the FFT pattern, the orientation of (002) spacing became anisotropic, and its intensity increased, proving the alignment of graphene sheets and the formation of crystallites. 
a
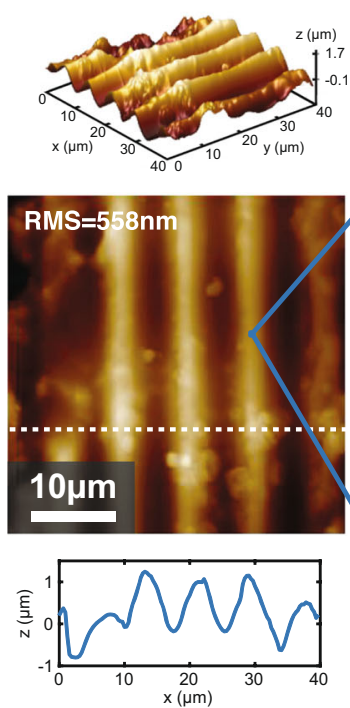

As-pyrolyzed
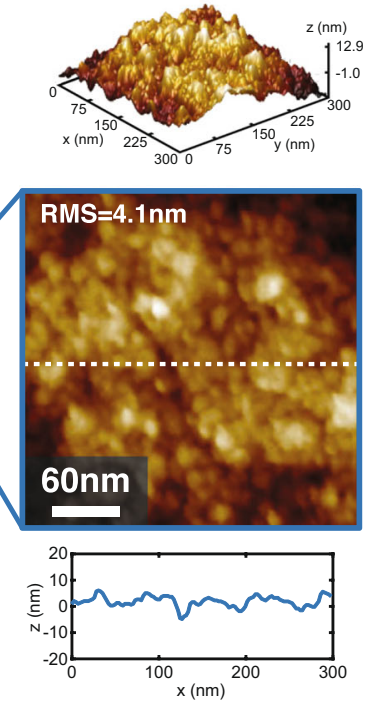

b
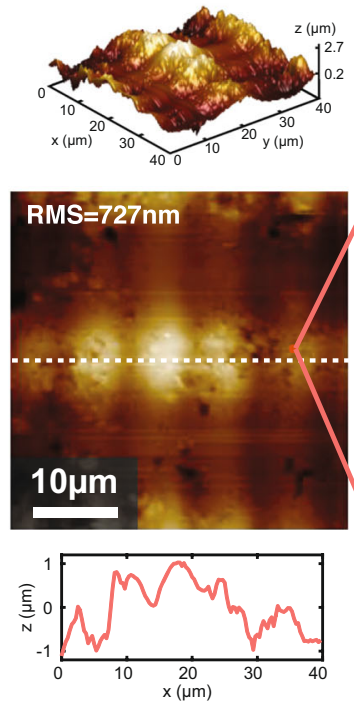

Joule-heated
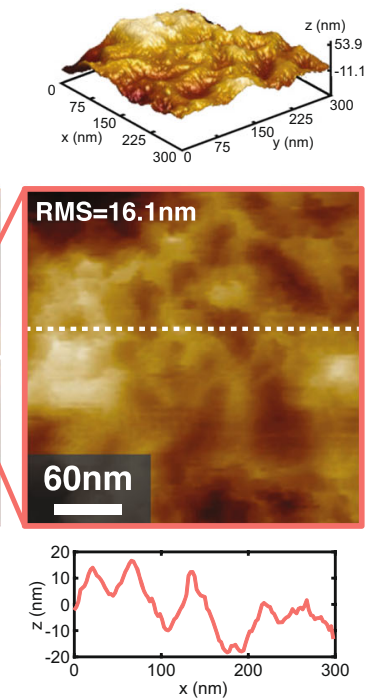

Fig. 2 Surface structural characterization with AFM. Scans of a representative as-pyrolyzed (a) and Joule-heated (b) carbon microlattice strut at the magnifications of $40 \mu \mathrm{m} \times 40 \mu \mathrm{m}$ and $300 \mathrm{~nm} \times 300 \mathrm{~nm}$. Each column contains a 3D image of the textured beam surface, its 2D representation, and the height profile extracted from the white dotted line in the 2D illustration. AFM images convey that Joule heating treatment enhanced surface roughness. From low magnification images, the root mean square (RMS) peak-to-valley groove depth increases from $558 \mathrm{~nm}$ for as-pyrolyzed sample to $727 \mathrm{~nm}$ for Joule-heated specimen. High magnification analyses reveal the development of homogeneously distributed nanopores introduced by Joule heating and testified by an RMS nanotopology that nearly quadrupled, rising from $4.1 \mathrm{~nm}$ in as-pyrolyzed sample to $16.1 \mathrm{~nm}$ in Joule-heated specimen.
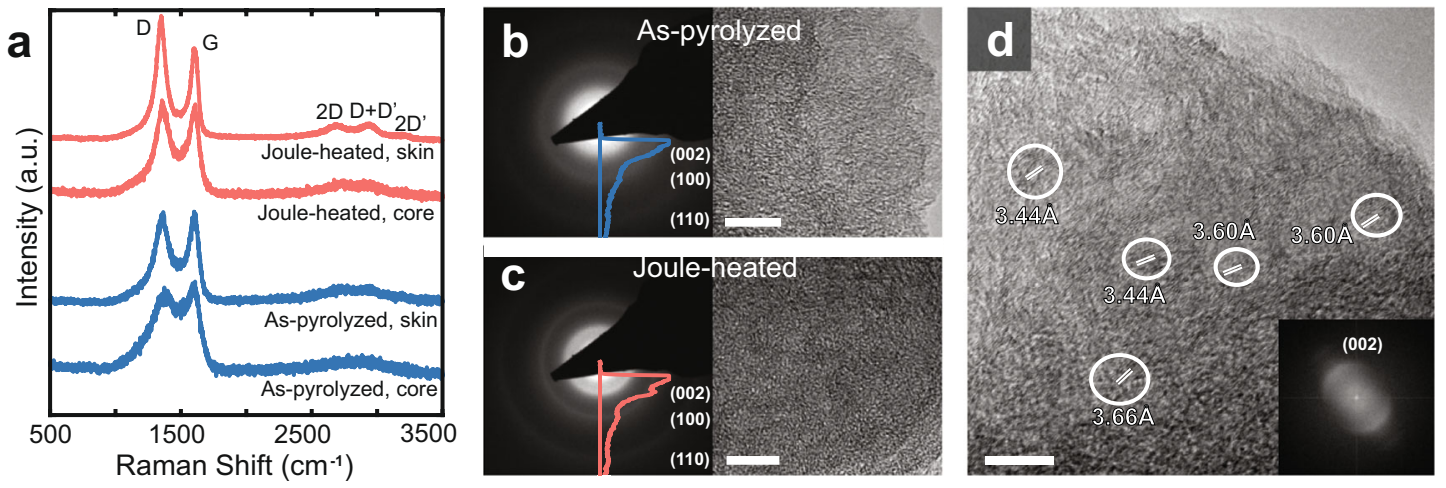

Fig. 3 Characterization of constituent carbon by Raman spectroscopy and TEM. a Raman spectra collected with an incident wavelength $\lambda=514 \mathrm{~nm}$ from the skin and the inner core of the beams reveal that the as-pyrolyzed samples are mainly constituted of amorphous carbon, while Joule heating increased the degree of $\mathrm{sp}^{2}$-hybridization and introduced a defect-rich nanographitic skin. Representative diffraction patterns and TEM images of as-pyrolyzed (b) and Joule-heated (c) carbon. Inset spectra with diffraction patterns indicate the grayscale intensity of diffraction rings for each crystallographic direction. d A high-resolution TEM (HRTEM) image of Joule-heated carbon containing nanocrystalline graphite and its FFT pattern. Circles highlight the nanocrystals that are $\sim 10 \mathrm{~nm}$ long, with graphene sheets spaced at $\sim 3.4-3.7 \AA$. The inset reports the intensity of the FFT diffraction ring for graphite (002), which increases along the direction where graphene sheets are stacked. Scale bars are $10 \mathrm{~nm}$.

The densification associated with graphitization of noncrystalline carbon, testified by the higher density of graphite, $2.23 \mathrm{~g} \mathrm{~cm}^{-3}$, than that of amorphous carbon, $1.8-2.0 \mathrm{~g} \mathrm{~cm}^{-3}$, was likely the cause of micro- and nanopores formation after Joule heating (Figs. 1 and 2). A similar roughening of the surface of carbon fibers by Joule heating has been reported, associated with graphitization of amorphous carbon ${ }^{48}$.

Contact angle measurements. The SLA-induced surface grooves, lattice architecture and Joule heating treatment contributed to increasing the water contact angle of additively manufactured carbon, which presents an inherently hydrophilic behavior, with $\theta=70-80^{\circ}$ (refs. ${ }^{49-51}$ ). The wettability was assessed on three types of 3D-printed carbon samples, namely as-pyrolyzed plates, as-pyrolyzed microarchitectures, and Joule-heated microlattices.

At the initial contact, the $0.7 \mu \mathrm{l}$ water droplet spontaneously spread over the surface of the as-pyrolyzed plates and microlattices. A different response was observed for Joule-heated microlattices, where the initial contact area was approximately one order of magnitude smaller than on the as-pyrolyzed samples (Fig. 4a and Supplementary Movie 1). At equilibrium, the contact interface areas were $1.70 \pm 0.31 \mathrm{~mm}^{2}$ for as-pyrolyzed plates, $1.14 \pm 0.25 \mathrm{~mm}^{2}$ for as-pyrolyzed microlattices, and $0.61 \pm$ $0.03 \mathrm{~mm}^{2}$ for Joule-heated microarchitectures.

The contact angles were measured along the parallel $\left(\theta_{/ /}\right)$and perpendicular $(\theta \perp)$ directions defined by the SLA-induced grooves (Fig. 4b and Supplementary Table 1). The presence of 
a

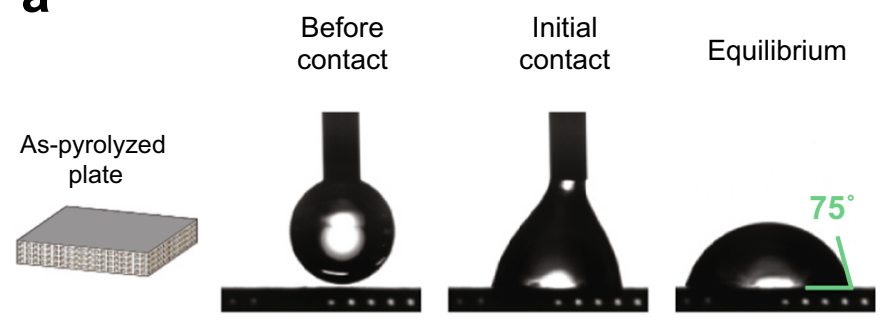

As-pyrolyzed microlattice

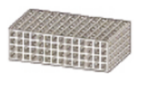

Joule-heated microlattice

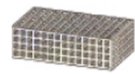

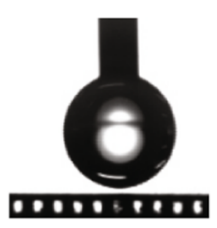
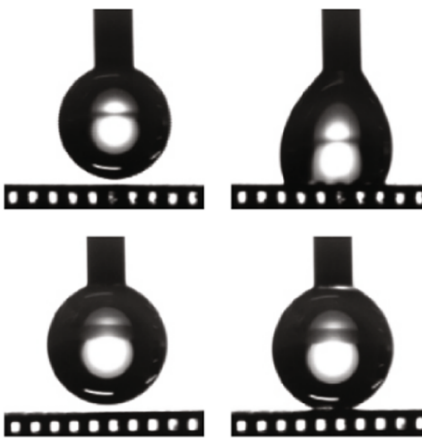

Before
contact

Initial
contact

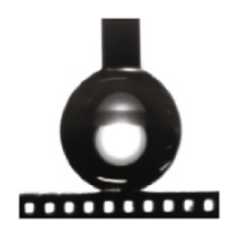

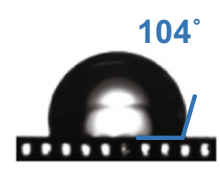

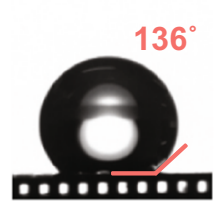

b

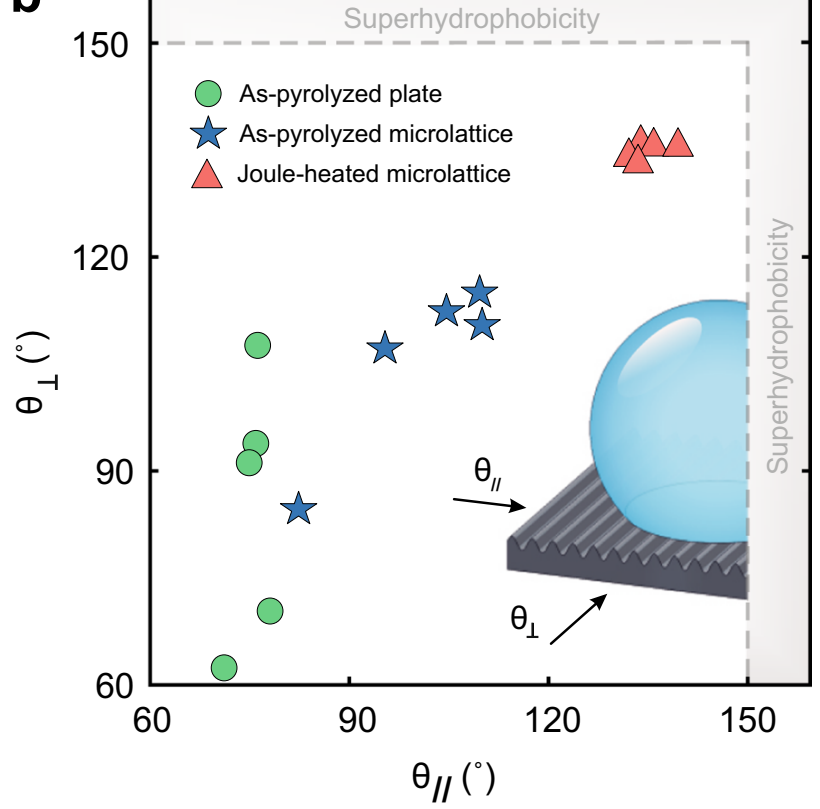

Fig. 4 Water contact angle measurements on pyrolytic and Joule-heated carbon. a Representative configurations of a $0.7 \mu l$ water droplet on an aspyrolyzed plate, as-pyrolyzed microlattice, and Joule-heated microarchitecture. Digital images were taken before contact, at the initial contact, and at equilibrium. The water contact angles were measured along the direction parallel to the SLA-induced grooves and prove that the lattice topology transforms pyrolytic carbon into a hydrophobic material, with Joule heating further enhancing the contact angle. b Water contact angles measured at equilibrium parallelly $\left(\theta_{/ /}\right)$and perpendicularly $(\theta \perp)$ to the groove direction, as shown in the inset. In Joule-heated microlattices, the water contact angle attains a tight distribution around $135^{\circ}$, approaching superhydrophobicity.

the grooves in carbon plates led to anisotropic contact angles, with $\theta_{/ /}=76 \pm 3^{\circ}$ and $\theta \perp=85 \pm 18^{\circ}$. The significant standard deviation of $\theta \perp$, as opposed to the tight distribution of $\theta_{/ /}$, suggests the presence of thermodynamically unstable airgaps between the droplet and the grooved surface. In as-pyrolyzed microarchitectures, the lattice structure introduced hydrophobicity through a Cassie-Baxter state, showing $\theta_{/ /}=100 \pm 12^{\circ}$ and $\theta \perp=106 \pm 12^{\circ}$. The microstructure also reduced the orientationdependent wetting response, proving that additive manufacturing can effectively modulate the water contact angle of architected materials.

A significant reduction of the wettability was observed in Jouleheated samples, where the contact angles increased to $\theta_{/ /}=135 \pm$ $3^{\circ}$ and $\theta \perp=135 \pm 1^{\circ}$. The average contact angles rose by $35^{\circ}$ and $29^{\circ}$ for parallel and perpendicular directions, respectively, thus exceeding the gain observed between as-pyrolyzed plate and aspyrolyzed microlattice. The increased hydrophobicity stemmed from the effects of the Joule heating treatment, which introduced a porous nanographitic skin. Highly $\mathrm{sp}^{2}$-hybridized carbon presents a contact angle higher than $90^{\circ}$ even in the absence of surface porosity ${ }^{52}$, improving the hydrophobic response of amorphous carbon. Moreover, the development of a surface porosity reduces the solid fraction of the water-carbon interface, governed by nanopores at a submicron scale. Therefore, the combination of $\mathrm{sp}^{2}$-hybridization with the development of a hierarchical lattice texture allowed both $\theta_{/ /}$and $\theta \perp$ to achieve a mean value of $135^{\circ}$ without discernable anisotropy. Although the present results were yet to achieve superhydrophobicity $\left(\theta>150^{\circ}\right)$, which could be obtained by increasing the power input for Joule heating that leads to higher degree of $\mathrm{sp}^{2}$-hybridization, they demonstrate a simple process to significantly increase the water contact angle without employing fluorination process ${ }^{53}$, plasma-enhanced chemical vapor deposition (PECVD) ${ }^{50}$ or nanoengineered coatings ${ }^{54,55}$.
Micro- and nanomechanical characterization. The mechanical properties of as-pyrolyzed and Joule-heated carbon microlattices were investigated through uniaxial compression experiments (Fig. 5a, b and Supplementary Movie 2). Eight specimens of each type of samples were compressed, with the average densities of $0.553 \pm 0.015 \mathrm{~g} \mathrm{~cm}^{-3}$ and $0.552 \pm 0.021 \mathrm{~g} \mathrm{~cm}^{-3}$, for as-pyrolyzed and Joule-heated microlattices, respectively. Engineering stressstrain data presented an initial toe region caused by misalignment, roughness and imperfection in the manufactured specimens. They revealed that all samples failed catastrophically and brittly at similar remarkable compressive stresses, with a significant variation in the compressive strength of the as-pyrolyzed samples, $151.26 \pm 54.53 \mathrm{MPa}$, and with a much tighter distribution for Joule-heated microlattices, $152.92 \pm 11.21 \mathrm{MPa}$ (Supplementary Table 2). The structural stiffness of each type of architectures was calculated from the linear elastic region of the stress-strain curves and resulted in $4.82 \pm 0.61 \mathrm{GPa}$ for the aspyrolyzed samples and $5.50 \pm 0.97 \mathrm{GPa}$ for the Joule-heated specimens. The different mechanical responses obtained for the two types of lattices became negligible on the core of the beam when subjected to nanoindentation tests (Fig. 5c). The Young's moduli $E_{\mathrm{c}}$ of core materials were calculated to be $25.38 \pm 2.75 \mathrm{GPa}$ for aspyrolyzed and $25.35 \pm 2.01 \mathrm{GPa}$ for Joule-heated samples (Supplementary Table 3), similar to Young's moduli of disordered carbon materials ${ }^{56,57}$.

Figure $5 \mathrm{~d}$ shows the material property map for compressive strength versus density of the developed carbon microlattices, together with various structural materials and high-strength truss micro-2,7,28-30,58-60 and nanolattices ${ }^{25,26,61}$. Compression experiments indicate that both types of microlattices fail brittly at virtually the same average stress of $\sim 152 \mathrm{MPa}$, which is stronger by one order of magnitude than the compressive strength of carbon aerogels ${ }^{58}$, and by a factor of $2-3$ than that of conventional structural materials, for the same density. The 
a

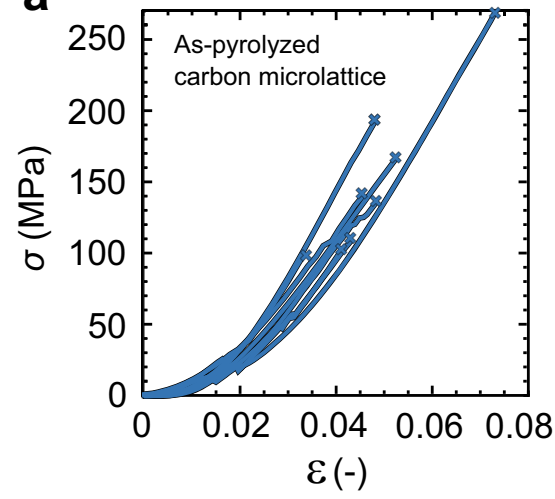

b

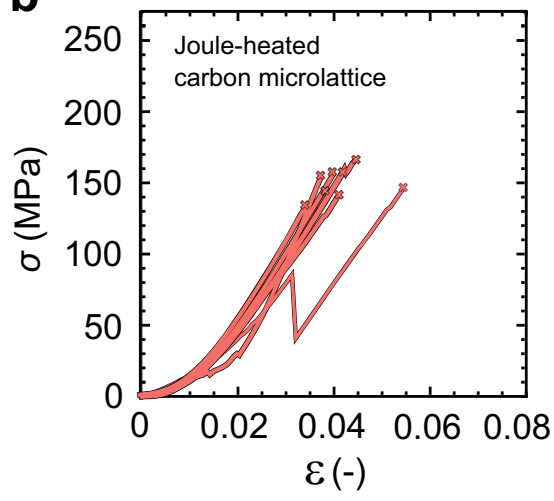

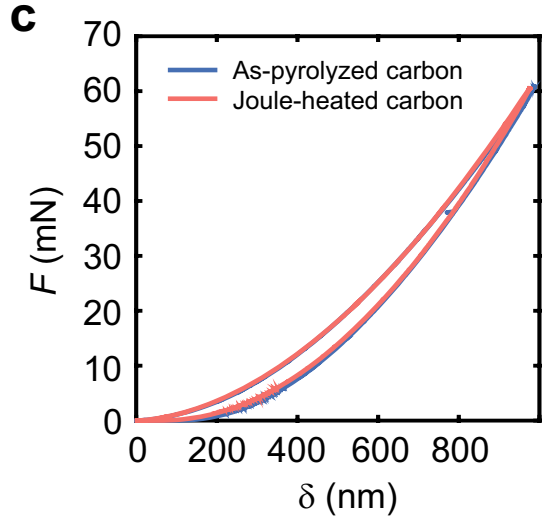

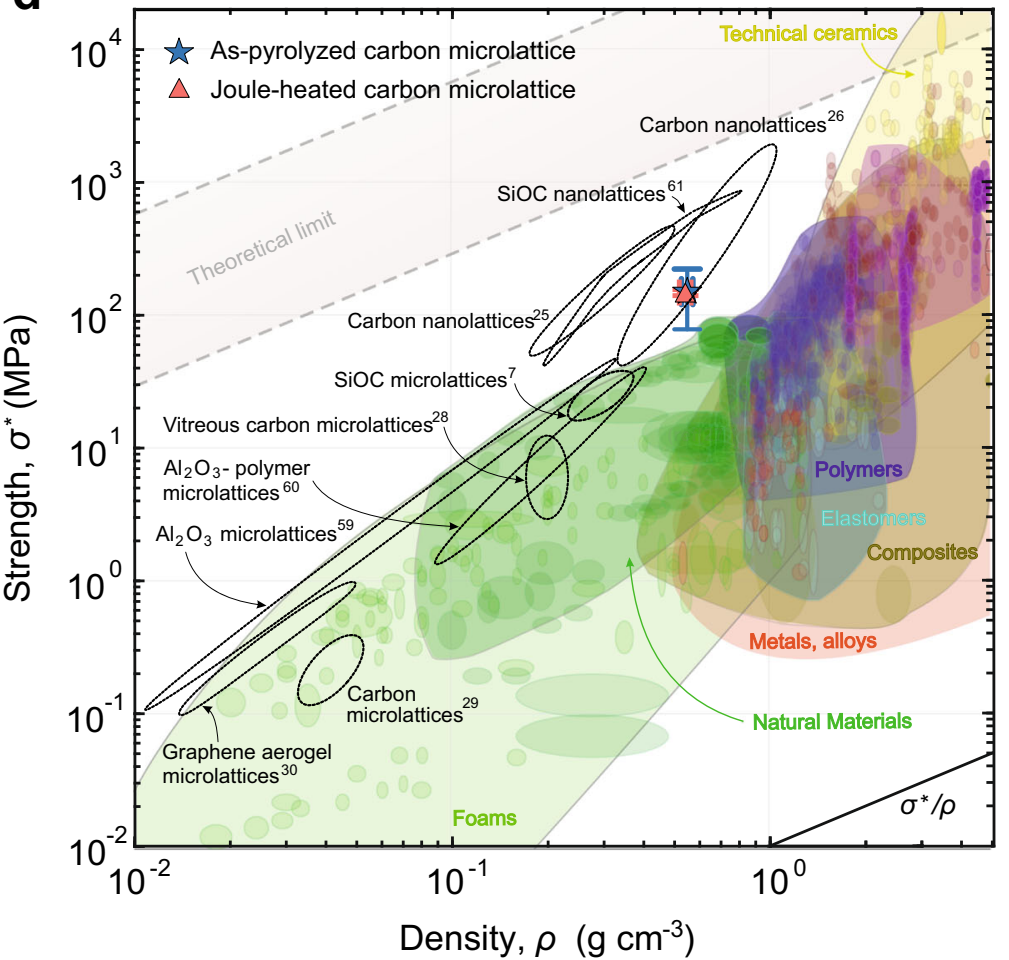

$\mathbf{e}$

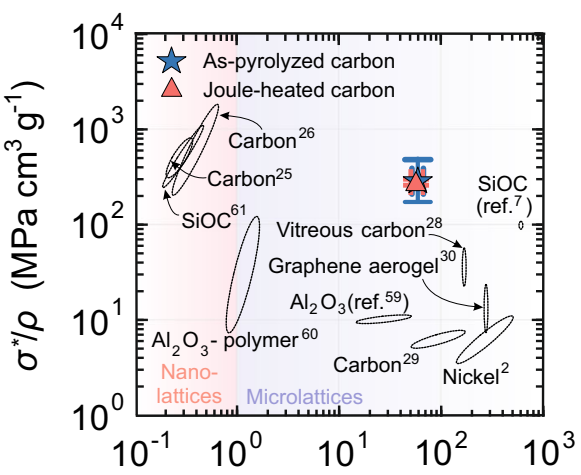

f

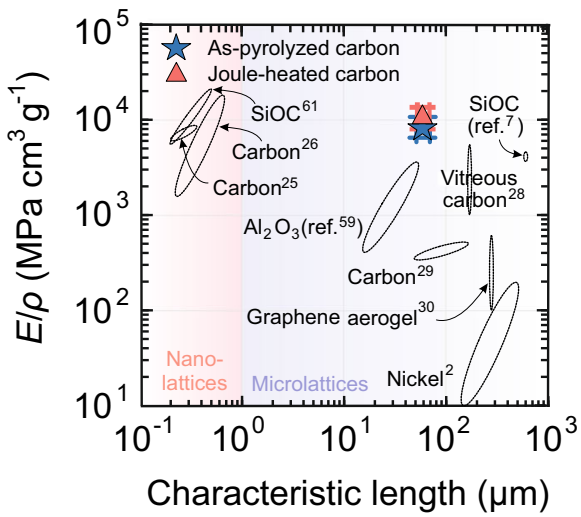

Fig. 5 Compressive mechanical properties for pyrolytic and Joule-heated carbon microlattices. Engineering stress-strain curves from micromechanical compression experiments on as-pyrolyzed (a) and Joule-heated (b) carbon microlattices. c Load versus displacement curves from nanoindentation tests performed on internal cores of beams. d Strength versus density plot of the fabricated microarchitectures in the context of structural materials (CES Edupack 2018, Granta Design) and high-strength micro- and nanolattices. Comparison of the specific strength (e) and specific stiffness (f) versus characteristic length (strut width) for micro- and nanoarchitectures. With two orders of magnitude larger strut diameter, the carbon lattices in this work attain properties that approach those of the strongest nanoarchitectures created up to date, outperforming all existing microlattices. Error bars indicate standard deviation.

strength achieved in this work represents the highest value ever recorded for microstructured solids, and approaches that of pyrolytic carbon ${ }^{25,26}$ and $\mathrm{SiOC}^{61}$ nanoarchitectures, which are the strongest truss lattices fabricated up to date, reaching up to $\sim 550 \mathrm{MPa}$, for the same density ${ }^{61}$.

Figure 5e, f reports the specific compressive strength and specific Young's modulus of the carbon microlattices in the context of high-strength micro- and nanoarchitectures, compared through their characteristic strut width. The plots convey that the fabricated carbon architectures possess specific strength and stiffness more than one order of magnitude higher than those of nickel $^{2}$, carbon ${ }^{29}$, graphene-aerogel ${ }^{30}$, and alumina ${ }^{59}$ microlattices, and 2-7 times greater than $\mathrm{SiOC}^{7}$, vitreous carbon ${ }^{28}$, and alumina coated polymer ${ }^{60}$ microlattices. Currently, only pyrolytic carbon and SiOC nanolattices synthesized by using two-photon lithography have similar or higher specific compressive properties, attainable through size effects at the strut width of hundreds of nanometers, which corresponds to the critical size for material flaw insensitivity ${ }^{25-27,61}$. Considering Griffith's law for brittle materials and assuming that the intrinsic length scale (critical flaw size, $a_{c}$ ) is on the same order as the extrinsic one (characteristic length of the lattice, $d$ ), the specific strength for lattices composed of the same constituent material scales as $\sigma^{*} \propto d^{-0.5}$. In contrast to strength, the stiffness of lattice architectures is not affected by size-effects, and the reduction of the length scale of micro and nanolattices has not been proven to enhance rigidity. The plot of the compressive strength normalized by the stiffness of the constituent material, reported as a function 
of the relative density for several high-strength lattices (Supplementary Fig. 7), further testifies the significant ultimate stress achieved by the carbon microarchitectures, outperforming all existing microlattices and approaching the strongest open-cell nanostructured materials ever realized. However, our microlattices present strut width and unit cell size two-orders of magnitude higher than those nanostructured solids. Therefore, the manufactured lattices prove that their remarkable strength and stiffness are compatible with a more scalable fabrication towards mesostructured solids. The unique mechanical properties of the fabricated microlattices are attributed to the combination of the manufacturing process and the anisotropic unit cell topology, where the former guarantees a high-quality pyrolytic carbon, while the latter ensures stiffness and strength through a high proportion of struts aligned with the loading direction ${ }^{62}$. Although some unit cell topologies were indicated as quasiisotropic in pioneering studies, recently it has been proven that almost all of the micro and nanoarchitectures considered in Fig. $5 \mathrm{~d}-\mathrm{f}$ are anisotropic ${ }^{63,64}$. The Zener ratio $a_{\mathrm{r}}$ depends on the relative density $\bar{\rho}$ and quantifies the degree of anisotropy for unit cells with cubic symmetry, where $a_{\mathrm{r}}=1$ corresponds to an isotropic configuration, while $a_{\mathrm{r}}<1$ and $a_{\mathrm{r}}>1$ indicate that the unit cell presents the highest stiffness along $\langle 100\rangle$ and $\langle 111\rangle$, respectively. Whilst the former response can be found in cubic unit cell and the latter in octet architectures, which represent the most investigated topology up to date, both geometries are anisotropic (Supplementary Fig. 8). The ideal chosen topology possess $a_{\mathrm{r}}=0.22$, while less anisotropic octet microlattices with $a_{\mathrm{r}}=1.56$ for a similar relative density and unit cell dimensions ${ }^{33}$, obtained through the presented manufacturing process, showed average stiffness and strength that were $\sim 50 \%$ and $~ 80 \%$ of that of the as-pyrolyzed microlattices, thus proving the advantages of the cubic topology over a stretching dominated geometry when employed in a non-slender lattice and subjected to preferential loading directions. Beyond open-cell three-dimensional lattices, although the design of architected metamaterials has relied on highly anisotropic geometries, as nano-honeycombs ${ }^{25}$, to achieve exceptional mechanical properties, very recent studies have theorized $^{65}$ and experimented ${ }^{66}$ almost isotropic architectures capable of achieving the Hashin-Shtrikman upper bound through closed-cell topologies governed by different deformation and failure mechanisms.

While the average failure stress remained unchanged after Joule heating, the standard deviation drastically reduced by $\sim 80 \%$. The heat treatment notably diminished the strength of the bestperforming as-pyrolyzed sample, but also increased the ultimate stress of the worst-performing as-pyrolyzed one. The reduced variability and the preserved average strength offered structural reliability, traditionally sought during the manufacturing process to improve the knowledge of mechanical properties and perform a probabilistic design with greater confidence ${ }^{67}$. The unchanged mean strength suggests that both types of microlattices globally failed via the same mechanism of catastrophic failure at the structural flaws within the material, and phenomenologically convey that Joule heating suppressed failure initiation at stresses significantly distant from the average failure stress. The combination of additive manufacturing and material reconfiguration through carbonization of polymeric precursor implies the development of various defects such as cracks, pores, and voids, all of which serve as locations of stress concentrations during mechanical loading (Supplementary Fig. 9). The stochastic signature of failure stresses in the as-pyrolyzed samples is assumed to be a result of a broad distribution of such flaws of different sizes and orientations throughout the microlattice. In Joule-heated samples, failure initiates at more tightly distributed surface flaws, significantly reducing the strength variability of as- pyrolyzed microlattices. On the one hand, the Joule heating process annealed the pre-existing critical flaws, preventing failure significantly below the average strength. On the other hand, it relieved the compressive residual stresses caused by the pyrolysis of the photoresists ${ }^{68}$, which can prevent crack propagation, and introduced surface defects, thus reducing the highest strengths recorded for as-pyrolyzed lattices. Therefore, the porous sheath is believed to contribute to the narrow deviation of compressive strength, suggesting surface nanoporosity as the preferred location where brittle failure is triggered.

This hypothesis is proven by the relationship between the critical semi-elliptical surface flaw size $a_{\mathrm{c}}$ and the failure strength of brittle material $\sigma_{\mathrm{f}}$, given by $a_{\mathrm{c}}=K_{\mathrm{IC}}{ }^{2} /\left(\pi \sigma_{\mathrm{f}}^{2}\right)$, where $K_{\mathrm{IC}}=0.91 \mathrm{MPa}{ }_{\mathrm{m}}$ is the carbon fracture toughness ${ }^{57,69}$. The bulk fracture strength of glassy carbon is size-dependent ${ }^{70}$, and it increases with a reduction of strut size because of the lower probability of finding a critical flaw. Assuming a fracture strength of $1.5-2.5 \mathrm{GPa}$, similarly to the values measured for pyrolytic glassy carbon fibers of $5 \mu \mathrm{m}$ diameter $^{71}$, the critical flaw size results $\sim 40-120 \mathrm{~nm}$, which corresponds to the dimension of the measured surface nanoporosity (Fig. 3).

Apart from its contribution to the reduced strength variability, Joule heating did not significantly improve structural properties: the average failure stress remained unchanged, and the mean lattice stiffness marked a moderate increase of $\sim 15 \%$. Other thermal processes, such as standard annealing at temperatures above $2000^{\circ} \mathrm{C}$, would be more effective than Joule-heating in improving mechanical features, although they would not guarantee a significant hydrophobic response. The porous nanographitic sheath is likely the cause of the increase in the average lattice stiffness from as-pyrolyzed specimens. The aspyrolyzed microlattices were assumed to be homogeneously constituted of non-crystalline pyrolytic carbon owning elastic modulus of $25.38 \mathrm{GPa}$ as from core nanoindentation, with negligible stiffness difference between the core and the skin of the beams. Finite element analysis of pyrolytic carbon microlattices predicted Young's modulus of $4.79 \mathrm{GPa}$ (Supplementary Fig. 10), consistent with the micromechanical experimental measurements. The SLA-induced corrugations were not included in the numerical model as simulations revealed that the effect of the bidirectional traces of SLA on stiffness was negligible (Supplementary Fig. 11). Nanoindentation on the internal core of Joule-heated samples revealed the same bulk stiffness as in aspyrolyzed specimens, while the beam texture prevented a reliable measurement of the elastic modulus of the skin. However, the porous nanocrystalline morphology of Joule-heated sheath suggested an increased stiffness when compared to that of the non-crystalline strut core ${ }^{39}$. Numerical analyses indicated that the homogenized Young's modulus of Joule-heated carbon increased to $29.19 \mathrm{GPa}$, a response attributed to the combined stiffening effects of the porosity ${ }^{72}$ and nanographitic layers ${ }^{73-75}$ that developed on the skin of Joule-heated microlattices.

\section{Discussion}

The paradigm for the development of lightweight metamaterials with superior mechanical performances has been the capitalization of strengthening size effects through the reduction of the lattice structural elements to the nanoscale, which inherently hinders a scalable fabrication. Furthermore, the simultaneous realization of a lightweight, strong, stiff, and hydrophobic single material has remained a challenge that prevents the application of these complementary properties in a crossfunctional solid.

We created a microarchitected carbon that combines excellent mechanical performances and remarkable hydrophobicity. Through a combination of SLA and pyrolysis, we manufactured 
carbon microlattices with strut width of $60-70 \mu \mathrm{m}$, cubic unit cell size of $\sim 200 \mu \mathrm{m}$ and density of $0.55 \mathrm{~g} \mathrm{~cm}^{-3}$. Although the unit cell topology was not optimized, the developed anisotropic architectures attained a specific strength up to $468.62 \mathrm{MPa} \mathrm{cm} \mathrm{g}^{-1}$, and a specific stiffness as high as $14.39 \mathrm{GPa} \mathrm{cm}^{3} \mathrm{~g}^{-1}$. The specific mechanical properties exceeded those of all existing meso- and microlattices, and they approached the stiffest and strongest nanoarchitectures realized so far ${ }^{25,26,61}$, albeit possessing unit cell dimensions two orders of magnitude larger and allowing faster creation of strong architectures. This work also demonstrated how to engender hydrophobicity in carbon-based materials through rational design of the lattice architecture, showing a facile technique to approach superhydrophobicity via Joule heatinginduced graphitization and development of a hierarchical lattice surface. Whilst the effect of the heat treatment predominantly altered the strut sheath, thus enabling noteworthy contact angles, it did not improve significantly the mechanical response, which arises from the bulk and could be further enhanced through other treatments. Nevertheless, we found Joule heating to preserve the mean strength while reducing its variability, and simultaneously increasing the lattice rigidity through a skin stiffened by porous nanographite. Compared to thermal treatment in a standard tube furnace, Joule heating can achieve higher temperatures, enables further graphitization, is faster and consumes less energy. However, the treatment might also induce undesired effects which, depending on the application, can hinder the mechanical response. This includes surface nanoporosity that could prevent the constituent carbon material from attaining the theoretical maximum strength, the removal of residual stresses which can cause the loss of the highest compressive strength values of aspyrolyzed carbon, and inhomogeneous heat distribution that could cause edge effects in large samples.

The combination of lightweight, strength, stiffness, and hydrophobicity makes nanographite-coated carbon microlattices potential candidates as a tunable multifunctional material for extreme environments, with applications ranging from water purification membranes ${ }^{76}$ to structural elements of vehicles where load-bearing and ice-inhibiting surfaces are required ${ }^{77}$.

\section{Methods}

Fabrication. Polymer lattices were prepared by $3 \mathrm{D}$ printing a transparent photoresist resin (PR-48) in a DLP SLA Amber Autodesk 3D printer, using a layer thickness of $25 \mu \mathrm{m}$. Each lattice had a periodic three-dimensional pattern of $600 \mu \mathrm{m}$-wide cubic unit cells with a beam width of $200 \mu \mathrm{m}$ and a length of $400 \mu \mathrm{m}$. Dimensions of specimens are reported below as thickness $\times$ width $\times$ length by the number of unit cells, where SLA layers were deposited along the width direction, and the bottom-most anchoring layer was designed to be thicker in order to sustain the microlattice during printing. Specimens for mechanical characterization were composed of $4 \times 8 \times 14$ unit cells. Specimens for contact angle measurements were designed to provide an area for a droplet of deionized (DI) water to deposit without reaching the edge of the lattices, containing $4 \times 10 \times 24$ unit cells. In order to assess the influence of the architected geometry on the water contact angle, plates were fabricated with an area equivalent to $20 \times 19$ unit cells. The additive manufactured samples were post-cured under sunlight for a day. The thicker anchoring layer was removed using a razor blade to prevent geometry distortion during pyrolysis, thus reducing by one the number of unit cells along the width of the samples.

The polymeric microlattices were pyrolyzed under vacuum of $<50 \mathrm{mtorr}$ in a $22 \mathrm{~mm}$ diameter fused quartz tube set in a Lindberg tube furnace, model 54357. During pyrolysis, the furnace temperature was differentially increased to yield fully dense carbon lattices and to prevent trapping of gasified components. The temperature was first elevated to $300^{\circ} \mathrm{C}$ and kept constant for $4 \mathrm{~h}$, then raised to $400^{\circ} \mathrm{C}$ and held constant for $1 \mathrm{~h}$, followed by the final carbonizing step at $1000^{\circ} \mathrm{C}$ for $4 \mathrm{~h}$, with all heating rates performed at $10^{\circ} \mathrm{C} \mathrm{min}^{-1}$.

After pyrolysis, the carbon microlattices were cooled down to room temperature, the vacuum was removed, and the samples were transferred to a cylindrical custommade apparatus (Supplementary Fig. 12), where Joule heating was performed. The as-pyrolyzed lattices were clamped between a pair of gold-plated copper electrodes that came in contact with the $4 \times 7$ unit cell surfaces and were suspended in a cylindrical glass jar. After purging the chamber with $100 \mathrm{~cm}^{3} \mathrm{~min}^{-1}$ of argon flow for a minute, we applied current for $10 \mathrm{~min}$ to produce a power density of
$10-12 \mathrm{~kW} \mathrm{~g}^{-1}$. The argon flow was maintained at $100-400 \mathrm{~cm}^{3} \mathrm{~min}^{-1}$, depending on the sample size, to preserve the integrity of the material.

Manufacturing imperfections against the original CAD model arose during additive fabrication. The strut width in the generated lattices was dependent on orientation, with $d_{1}$ and $d_{2}$ that identify the beams deposited parallelly or perpendicularly to the printer platform, while $l$ represents the unit cell size. Therefore, the microlattices relative density $\bar{\rho}$ is formulated as

$$
\bar{\rho}=\frac{2 d_{1}^{2}+d_{2}^{2}}{l^{2}}-\frac{d_{1} d_{2}^{2}+d_{2}^{3}}{l^{3}},
$$

where the first and second terms account for the beam and node relative density, respectively.

The relative density was calculated for three different samples of both types of lattices by averaging the measured characteristic lengths, providing $\bar{\rho}=0.30 \pm 0.01$ for as-pyrolyzed and $\bar{\rho}=0.28 \pm 0.01$ for Joule-heated microlattices.

Material characterization. Atomic-level microstructures of the lattices before and after Joule heating treatment were investigated using Raman spectroscopy (Renishaw M1000 Micro Raman Spectrometer System), SEM (Thermo-Fisher Versa 3D DualBeam), Transmission Electron Microscopy (TEM, Thermo-Fisher, Tecnai TF-30), and AFM (Brucker Dimension Icon). Raman spectra were collected with a green laser (wavelength $\lambda=514 \mathrm{~nm}$ ). The internal core of the beam was inspected through Raman laser by removing $2 \mu \mathrm{m}$ of the external surface with an abrasive sheet containing $0.3 \mu \mathrm{m}$ alumina powder (Buehler, FiberMet Abrasive Discs). SEM and TEM samples were prepared by cleaving the carbon microlattices, collecting pieces of the generated debris, manually grinding them between two glass slides, and transferring the ground powder onto a copper TEM grid using carbon support (Pacific Grid Tech). The debris of specimens failed during micromechanical testing were analyzed with SEM, showing that the core of the beams was fully dense, while micropores developed only on the surface. AFM PeakForce Tapping (PFT) mode was used to characterize the surface roughness of each sample at three different locations by using a silicon probe with a tip radius $r_{\text {tip }}=$ $2 \mathrm{~nm}$ (Brucker SCANASYST-AIR). AFM scans were obtained on $40 \mu \mathrm{m} \times 40 \mu \mathrm{m}$ regions at a frequency of $0.1 \mathrm{~Hz}$, and on $300 \mathrm{~nm} \times 300 \mathrm{~nm}$ areas at a rate of $2 \mathrm{~Hz}$.

Contact angle measurements. The water contact angle was measured by a contact angle goniometer (Kyowa Kaimen Kagaku DM-301) equipped with a PTFE-coated 28 -gauge needle. Samples were mounted onto a movable stage with a $9 \times 24$ unit cell surface (microlattices) or $19 \times 19$ unit cell surface (plates) facing toward the needle. The stage was elevated until the sample entered in contact with the $0.7 \mu \mathrm{l}$ droplet of DI water (diameter $>500 \mu \mathrm{m})$ dispensed from the needle. Subsequently, the stage was retracted to detach the droplet from the syringe (Supplementary Movie 1). The contact angles were measured at equilibrium along the parallel $\left(\theta_{/ /}\right)$and perpendicular $(\theta \perp)$ directions defined by the SLA-induced grooves. The evaporation time of dispensed water droplets was recorded, showing a decrease of $\sim 20 \%$ from monolithic plates to microlattices. Five measurements per sample were conducted, in a laboratory environment with a temperature of $27^{\circ} \mathrm{C}$ and $60 \%$ relative humidity.

Nanomechanical and micromechanical characterization. Nanoindentation was performed on lattice nodes using a nanoindenter (Agilent, G200) equipped with a diamond Berkovich indenter tip. As-pyrolyzed and Joule-heated core samples were mounted on an SEM stub using a graphite paste, and they were indented to a depth of $1 \mu \mathrm{m}$ at the loading and unloading rates of $2 \mathrm{mN} \mathrm{s}^{-1}$. From the loaddisplacement curves, the Young's modulus $E_{\mathrm{c}}$ was calculated as ${ }^{78}$

$$
E_{\mathrm{c}}=\frac{1-\nu_{\mathrm{s}}^{2}}{\frac{1}{E_{\mathrm{r}}}-\frac{1-\nu_{\mathrm{i}}^{2}}{E_{\mathrm{i}}}},
$$

where $E_{\mathrm{r}}=\mathrm{S} \sqrt{\pi} /(2 \beta \sqrt{A})$ is the reduced Young's modulus, $S$ is the gradient of the load-displacement curve at the maximum depth of indentation, $A=24.675 h_{\mathrm{c}}^{2}+$ $0.562 h_{\mathrm{c}}+0.003216$ is the indentation area for the Berkovich indenter tip, $h_{\mathrm{c}}=$ $1 \mu \mathrm{m}$ is the depth of indentation, $\beta=1.081$ is the constant for the Berkovich indenter tip, $E_{\mathrm{i}}=1143 \mathrm{GPa}$ is the Young's modulus of the diamond indenter ${ }^{79}$, $v_{\mathrm{i}}=0.0691$ and $v_{\mathrm{s}}=0.21$ are the Poisson's ratios of the diamond indenter ${ }^{79}$ and pyrolytic carbon specimen ${ }^{80}$, respectively. For micromechanical characterization, compression tests on all microlattices were carried out on an electromechanical testing frame (Instron, 5569). The load was applied at a nominal rate of $2.5 \mu \mathrm{m} \mathrm{s}^{-1}$ on the $7 \times 14$ unit cells sample surface until failure (Supplementary Movie 2), while the displacement was measured with a laser extensometer (Electronic Instrument Research, LE-01) interfaced with the electromechanical apparatus for data synchronization. Eight specimens for both as-pyrolyzed and Joule-heated samples were compressed, with the load-displacement curve obtained. The engineering stress-strain curve was calculated by normalizing the measured force by the lattice cross-section and the compressive displacement by the sample height (Fig. 5a, b and Supplementary Table 2). The microlattice Young's modulus was determined from the linear-elastic portion of the stress-strain curve after the initial toe region, whereas the compressive strength represents the maximum stress achieved before brittle failure. 
Finite element analysis. The microlattice geometry obtained from SEM images was generated with SolidWorks 2018 (Dassault Systèmes) and imported in the finite element software Abaqus 2018 (Dassault Systèmes). The lattice was discretized with 1.2 million tetrahedral elements with Young's modulus $E_{\mathrm{c}}=25.38$ $\mathrm{GPa}$ measured through nanoindentation, and Poisson ratio $v=0.21\left(\right.$ ref. $\left.{ }^{80}\right)$. The as-pyrolyzed microlattices were assumed to be entirely constituted of amorphous pyrolytic carbon possessing uniform stiffness $E_{c}$. Starting from the experimentally measured average effective stiffness of the Joule-heated lattices, the homogenized Young's modulus of the Joule-heated carbon was calculated through numerical analysis as $29.19 \mathrm{GPa}$ (Supplementary Fig. 10), where the increase in elastic modulus is attributed to the stiffening effect of the $\sim 1-1.5 \mu \mathrm{m}$ thick porous nanographitic skin ${ }^{72-75}$. Displacement was imposed on the microlattice top surface while the resultant force was acquired, with boundary conditions imposed on the bottom surface. The lattice stiffness was obtained from the engineering stress-strain curve calculated through the compressive force-displacement relationship.

In order to assess the influence of SLA-induced corrugations, finite element simulations were performed on as-pyrolyzed uncorrugated and corrugated unit cells, showing that the effective stiffness varied by only $3.52 \%$ (Supplementary Fig. 11), thus justifying the choice of neglecting the bidirectional SLA traces in the full computational model. The topology of the corrugation was acquired from SEM images, generated with SolidWorks 2018 and imported in Abaqus 2018.

The quantification of the degree of anisotropy for unit cells with cubic symmetry was obtained through the Zener ratio $a_{\mathrm{r}}=\frac{2 C_{44}}{C_{11}-C_{12}}$, where $C_{\mathrm{ij}}$ represents the non-null components of the forth-order elastic stiffness tensor, written in Voigt notation. The Zener ratio of the ideal cubic topology was calculated through finite element simulations, and compared with that computed for the octet geometry, which represents the most investigated topology $25,26,33,59,61$, for relative densities $\bar{\rho}$ lower than $40 \%$. For each relative density, two analyses were sufficient to define $a_{\mathrm{r}}$. The first allows obtaining $C_{11}$ and $C_{12}$ by imposing a normal strain along $\langle 100\rangle$ to a laterally constrained unit cell, while the second gives $C_{44}$ when shear strains are applied to two adjacent faces of the unit cell ${ }^{81}$.

\section{Data availability}

The main data that support the findings of this study are available within the Article and its Supplementary Information. Additional data are available from the authors on reasonable request.

Received: 13 October 2019; Accepted: 13 September 2020; Published online: 09 October 2020

\section{References}

1. Sachs, E., Cima, M. \& Cornie, J. Three-dimensional printing: rapid tooling and prototypes directly from a CAD model. CIRP Ann. - Manuf. Technol. 39, 201-204 (1990).

2. Schaedler, T. A. et al. Ultralight metallic microlattices. Science 334, 962-965 (2011).

3. Martin, J. H. et al. 3D printing of high-strength aluminium alloys. Nature. 549, 365-369 (2017).

4. Vyatskikh, A. et al. Additive manufacturing of 3D nano-architected metals. Nat. Commun. 9, 593 (2018)

5. Leung, C. L. A. et al. In situ X-ray imaging of defect and molten pool dynamics in laser additive manufacturing. Nat. Commun. 9, 1355 (2018).

6. Hagedorn, Y., Meiners, W., Wissenbach, K. \& Wilkes, J. Additive manufacturing of $\mathrm{ZrO} 2-\mathrm{Al} 2 \mathrm{O} 3$ ceramic components by selective laser melting. Rapid Prototyp. J. 19, 51-57 (2013).

7. Eckel, Z. C. et al. Additive manufacturing of polymer-derived ceramics Science 351, 58-62 (2016).

8. Kotz, F. et al. Three-dimensional printing of transparent fused silica glass. Nature 544, 337-339 (2017).

9. Nguyen, D. T. et al. 3D-printed transparent glass. Adv. Mater. 29, 1701181 (2017).

10. Meza, L. R. et al. Reexamining the mechanical property space of threedimensional lattice architectures. Acta Mater. 140, 424-432 (2017).

11. Zhang, X. et al. Three-dimensional high-entropy alloy-polymer composite nanolattices that overcome the strength-recoverability trade-off. Nano Lett. 18, 4247-4256 (2018).

12. Maggi, A., Li, H. \& Greer, J. Three-dimensional nano-architected scaffolds with tunable stiffness for efficient bone tissue growth. Acta Biomater. 63, 294-305 (2017).

13. Murphy, S. V. \& Atala, A. 3D bioprinting of tissue and organs. Nat. Biotechnol. 32, 773-785 (2014).

14. Zhu, C. et al. Supercapacitors based on three-dimensional hierarchical graphene aerogels with periodic macropores. Nano Lett. 16, 3448-3456 (2016).
15. Vak, D. et al. 3D printer based slot-die coater as a lab-to-fab translation tool for solution-processed solar cells. Adv. Energy Mater. 5, 1401539 (2015).

16. Wang, Q. et al. Lightweight mechanical metamaterials with tunable negative thermal expansion. Phys. Rev. Lett. 117, 175901 (2016).

17. Krodel, S. \& Daraio, C. Microlattice metamaterials for tailoring ultrasonic transmission with elastoacustic hybridization. Phys. Rev. Appl. 6, 064005 (2016).

18. Bertoldi, K., Vitelli, V., Christensen, J. \& van Hecke, M. Flexible mechanical metamaterials. Nat. Rev. Mat. 2, 17066 (2017).

19. Bauer, J. et al. Nanolattices: an emerging class of mechanical metamaterials. Adv. Mater. 29, 1701850 (2017).

20. Zhang, X., Wang, Y., Ding, B. \& Li, X. Design, fabrication, and mechanics of 3D micro-/nanolattices. Small 16, 1902842 (2019).

21. Greer, J. \& Deshpande, V. S. Three-dimensional architected materials and structures: design, fabrication, and mechanical behavior. MRS Bull. 44, 750-757 (2019)

22. Schwaiger, R., Meza, L. R. \& Li, X. The extreme mechanics of micro- and nanoarchitected materials. MRS Bull. 44, 758-765 (2019).

23. Meza, L. R., Das, S. \& Greer, J. R. Strong, lightweight, and recoverable threedimensional ceramic nanolattices. Science 345, 1322-1326 (2014).

24. Meza, L. R. et al. Resilient 3D hierarchical architected metamaterials. Proc. Natl Acad. Sci. USA 112, 11502-11507 (2015).

25. Bauer, J., Schroer, A., Schwaiger, R. \& Kraft, O. Approaching theoretical strength in glassy carbon nanolattices. Nat. Mater. 15, 438-443 (2016).

26. Zhang, X., Vyatskikh, A., Gao, H., Greer, J. R. \& Li, X. Lightweight, flawtolerant, and ultrastrong nanoarchitected carbon. Proc. Natl Acad. Sci. USA 116, 6665-6672 (2019).

27. Gao, H., Ji, B., Jager, I. L., Arzt, E. \& Fratzl, P. Materials become insensitive to flaws at nanoscale: lesson from nature. Proc. Natl Acad. Sci. USA 100, 5597-5600 (2003).

28. Jacobsen, A. J., Mahoney, S., Carter, W. B. \& Nutt, S. Vitreous carbon microlattice structures. Carbon 49, 1025-1032 (2011).

29. Chen, X. et al. Cellular carbon microstructures developed by using stereolithography. Carbon 123, 34-44 (2017).

30. Zhu, C. et al. Highly compressible 3D periodic graphene aerogel microlattices. Nat. Commun. 6, 6962 (2015)

31. Meija, R. et al. Nanomechanics of individual aerographitetetrapods. Nat. Commun. 8, 14982 (2016)

32. Guo, F. et al. Highly stretchable carbon aerogels. Nat. Commun. 9, 881 (2018)

33. Kudo, A., Misseroni, D., Wei, Y. \& Bosi, F. Compressive response of nonslender octet carbon microlattices. Front. Mater. 6, 169 (2019).

34. Wang, C., Jia, G., Taherabadi, L. H. \& Madou, M. J. A novel method for the fabrication of high-aspect ratio C-MEMS structures. J. Microelectromech. Syst. 14, 348-358 (2005)

35. Jung, S., Tiwari, M. K., Doan, N. V. \& Poulikakos, D. Mechanism of supercooled droplet freezing on surfaces. Nat. Commun. 3, 615 (2012).

36. Blossey, R. Self-cleaning surfaces-virtual realities. Nat. Mater. 2, 301-306 (2003).

37. Jafari, R., Cloutier, C., Allahdini, A. \& Momen, G. Recent progress and challenges with $3 \mathrm{D}$ printing of patterned hydrophobic and superhydrophobic surfaces. Int. J. Adv. Manuf. Technol. 103, 1225-1238 (2019).

38. Wang, $X$. et al. i3DP, a robust $3 \mathrm{D}$ printing approach enabling genetic postprinting surface modification. Chem. Commun. 49, 10064-10066 (2013).

39. Huang, Y. \& Young, R. J. Effect of fibre microstructure upon the modulus of PAN- and pitch-based carbon fibres. Carbon 33, 97-107 (1995).

40. Sadezky, A., Muckenhuber, H., Grothe, H., Niessner, R. \& Pöschl, U. Raman microspectroscopy of soot and related carbonaceous materials: Spectral analysis and structural information. Carbon 43, 1731-17 (2005).

41. Fishlock, S. J., Grech, D., McBride, J. W., Chong, H. M. H. \& Pu, S. H. Mechanical characterisation of nanocrystalline graphite using micromechanical structures. Microelectron. Eng. 159, 184-189 (2016).

42. Cuozi, M., Bruneel, J. L., Talaga, D. \& Bokobza, L. A multi wavelength Raman scattering study of defective graphitic carbon materials: The first order Raman spectra revisited. Carbon 107, 388-394 (2016).

43. Hargreaves, N. J. \& Cooper, S. Nanographite synthesized from acidified sucrose microemulsions under ambient conditions. Cryst. Growth Des. 16, 3133-3142 (2016).

44. Jerng, S. K. et al. Nanocrystalline graphite growth on sapphire by carbon molecular beam epitaxy. J. Phys. Chem. C 115, 4491-4494 (2011).

45. Pimenta, M. A. et al. Studying disorder in graphite-based systems by Raman spectroscopy. Phys. Chem. Chem. Phys. 9, 1276-1290 (2007).

46. Lee, Y. J. The second order Raman spectroscopy in carbon crystallinity. J. Nucl. Mater. 325, 174-179 (2004).

47. Ferrari, A. C. \& Robertson, J. Resonant Raman spectroscopy of disordered, amorphous, and diamondlike carbon. Phys. Rev. B 64, 075414 (2001).

48. Yao, Y. et al. Carbon welding by ultrafast Joule heating. Nano Lett. 16, 7282-7289 (2016) 
49. Mattia, D., Bau, H. H. \& Gogotsi, Y. Wetting of CVD carbon films by polar and nonpolar liquids and implications for carbon nanopipes. Langmuir 22, 1789-1794 (2006).

50. Yan, A., Xiao, X., Külaots, I., Sheldon, B. W. \& Hurt, R. H. Controlling water contact angle on carbon surfaces from $5^{\circ}$ to $167^{\circ}$. Carbon 44, 3116-3120 (2006).

51. Zhou, Y. et al. Control over the wettability of amorphous carbon films in a large range from hydrophilicity to super-hydrophobicity. Appl. Surf. Sci. 253, 2690-2694 (2006).

52. Rafiee, J. et al. Wetting transparency of graphene. Nat. Mater. 11, 217-222 (2012).

53. Feng, J., Qin, Z. \& Yao, S. Factors affecting the spontaneous motion of condensate drops on superhydrophobic copper surfaces. Langmuir $\mathbf{2 8}$, 6067-6075 (2012).

54. Lau, K. K. S. et al. Superhydrophobic carbon nanotube forests. Nano Lett. 3, 1701-1705 (2003).

55. Peng, C., Chen, Z. \& Tiwari, M. K. All-organic superhydrophobic coatings with mechanochemical robustness and liquid impalement resistance. Nat. Mater. 17, 355-360 (2018)

56. Wang, R. J. et al. Ultrasonic investigation of amorphous carbon in various frequencies at low temperatures from 2.1 to $300 \mathrm{~K}$. Solid State Commun. 127, 47-50 (2003).

57. Zhao, J. X., Bradt, R. C. \& Walker, P. L. The fracture toughness of glassy carbons at elevated temperatures. Carbon 23, 15-18 (1985).

58. Fairén-Jiménez, D., Carrasco-Marín, F. \& Moreno-Castilla, C. Adsorption of benzene, toluene, and xylenes on monolithic carbon aerogels from dry air flows. Langmuir 23, 10095-10101 (2007).

59. Zheng, X. et al. Ultralight, ultrastiff mechanical metamaterials. Science 344, 1373-1377 (2014).

60. Bauer, J., Hengsbach, S., Tesari, I., Schwaiger, R. \& Kraft, O. High-strength cellular ceramic composites with 3D microarchitecture. Proc. Natl Acad. Sci. USA 111, 2453-2458 (2014).

61. Bauer, J. et al. Additive manufacturing of ductile, ultrastrong polymer-derived nanoceramics. Matter 1, 1547-1556 (2019).

62. Egan, P. F., Gonella, V. C., Engensperger, M., Ferguson, S. J. \& Shea, K. Computationally designed lattices with tuned properties for tissue engineering using 3D printing. PLoS ONE 12, e0182902 (2017).

63. Chen, W. et al. Stiff isotropic lattices beyond the Maxwell criterion. Sci. Adv. 5, eaaw1937 (2019).

64. Patil, G. U. \& Matlac, K. H. Effective property evaluation and analysis of three-dimensional periodic lattices and composites through Bloch-wave homogenization. J. Acoust. Soc. Am. 145, 1259-1269 (2019).

65. Berger, J. B., Wadley, H. N. G. \& McMeeking, R. M. Mechanical metamaterials at the theoretical limit of isotropic elastic stiffness. Nature 543, 533-537 (2017).

66. Crook, C. et al. Plate-nanolattices at the theoretical limit of stiffness and strength. Nat. Commun. 11, 1579 (2020).

67. Todinov, M. Reliability and Risk Models: Setting Reliability Requirements. (John Wiley \& Sons, 2015)

68. Quang, L. N., Larsen, P. E., Boisen, A. \& Keller, S. Tailoring stress in pyrolytic carbon for fabrication of nanomechanical string resonators. Carbon 133, 358-368 (2018).

69. Brezny, R. \& Green, D. J. The effect of cell size on the mechanical behavior of cellular materials. Acta Metall. Mater. 38, 2517-2526 (1990).

70. Bullock, R. \& Kaae, J. Size effect on the strength of glassy carbon. J. Mater. Sci. 14, 920-930 (1979).

71. Kawamura, K. \& Jenkins, G. M. A new glassy carbon fibre. J. Mater. Sci. 5, 262-267 (1970).

72. Duan, H. L., Wang, J., Karihaloo, B. L. \& Huang, Z. P. Nanoporous materials can be made stiffer than non-porous counterparts by surface modification. Acta Mater. 54, 2983-2990 (2006)

73. Zhou, G., Liu, Y., He, L., Guo, Q. \& Ye, H. Microstructure difference between core and skin of T700 carbon fibers in heat-treated carbon/carbon composites. Carbon 49, 2883-2892 (2011).

74. Manoharan, M., Lee, H., Rajagopalan, R., Foley, H. C. \& Haque, M. A. Elastic properties of 4-6 nm-thick glassy carbon thin films. Nanoscale Res. Lett. 5, 14-19 (2010).
75. Tanaka, F., Okabe, T., Okuda, H., Kinloch, I. A. \& Young, R. J. The effect of nanostructure upon the compressive strength of carbon fibres. J. Mater. Sci. 48, 2104-2110 (2013).

76. Dongare, P. D. et al. Nanophotonics-enabled solar membrane distillation for off-grid water purification. Proc. Natl Acad. Sci. USA 114, 6936-6941 (2017).

77. Verho, T. et al. Mechanically durable superhydrophobic surfaces. Adv. Mater 23, 673-678 (2011)

78. Sakharova, N. A., Fernandes, J. V., Antunes, J. M. \& Oliveira, M. C. Comparison between Berkovich, Vickers and conical indentation tests: a three-dimensional numerical simulation study. Int. J. Solids Struct. 46, 1095-1104 (2009).

79. Klein, C. A. \& Cardinale, G. F. Young's modulus and Poisson's ratio of CVD diamond. Diam. Relat. Mater. 2, 918-923 (1993).

80. Price, R. J. \& Kaae, J. L. Poisson's ratio of pyrolytic carbon. Carbon 7, 706-708 (1969).

81. Xu, S., Shen, J., Zhou, S., Huang, X. \& Xie, Y. M. Design of lattice structures with controlled anisotropy. Mater. Des. 93, 443-447 (2016).

\section{Acknowledgements}

A.K. gratefully acknowledges the financial support from the Resnick Sustainability Institute at the California Institute of Technology and from Prof. Julia R. Greer (Caltech) through the Vannevar-Bush Faculty Fellowship of the US Department of Defense. The authors thank Prof. Julia R. Greer's research group (Caltech), Prof. Sergio Pellegrino (Caltech) and Prof. George Rossman (Caltech) for their support in conducting experiments in their laboratories, Prof. Julia R. Greer and Prof. Manish K. Tiwari (UCL) for useful discussions. A.K. thanks Research Promotion and Support Organization (Tohoku University). F.B. acknowledges the support from The Royal Society (RGS-R1191459).

\section{Author contributions}

A.K. and F.B. designed the research, discussed the results, wrote the paper, and commented on the manuscript. A.K. manufactured the specimens, performed contact angle experiments, material and nanomechanical characterization. A.K. and F.B. performed micromechanical tests. F.B. carried out the numerical analyses.

\section{Competing interests}

The authors declare no competing interests.

\section{Additional information}

Supplementary information is available for this paper at https://doi.org/10.1038/s43246020-00073-3.

Correspondence and requests for materials should be addressed to F.B.

Reprints and permission information is available at http://www.nature.com/reprints

Publisher's note Springer Nature remains neutral with regard to jurisdictional claims in published maps and institutional affiliations.

Open Access This article is licensed under a Creative Commons Attribution 4.0 International License, which permits use, sharing, adaptation, distribution and reproduction in any medium or format, as long as you give appropriate credit to the original author(s) and the source, provide a link to the Creative Commons license, and indicate if changes were made. The images or other third party material in this article are included in the article's Creative Commons license, unless indicated otherwise in a credit line to the material. If material is not included in the article's Creative Commons license and your intended use is not permitted by statutory regulation or exceeds the permitted use, you will need to obtain permission directly from the copyright holder. To view a copy of this license, visit http://creativecommons.org/ licenses/by/4.0/.

(C) The Author(s) 2020 